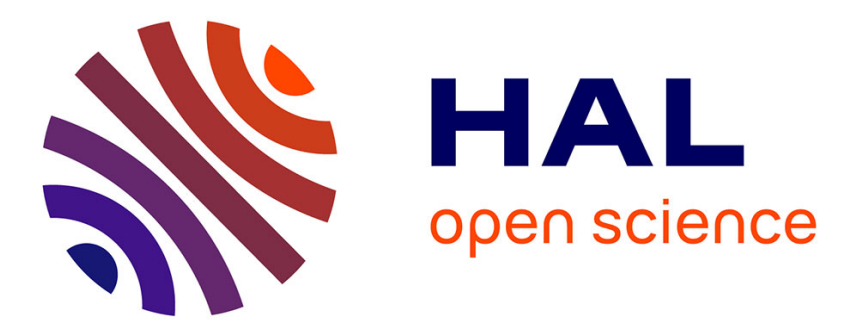

\title{
Lyapunov event-triggered control: a new event strategy based on the control
}

\author{
Nicolas Marchand, John Jairo Martinez Molina, Sylvain Durand, Fermi \\ Guerrero-Castellanos
}

\section{- To cite this version:}

Nicolas Marchand, John Jairo Martinez Molina, Sylvain Durand, Fermi Guerrero-Castellanos. Lyapunov event-triggered control: a new event strategy based on the control. NOLCOS 2013 - 9th IFAC Symposium on Nonlinear Control Systems, Sep 2013, Toulouse, France. hal-00828391

\section{HAL Id: hal-00828391 \\ https://hal.science/hal-00828391}

Submitted on 30 May 2013

HAL is a multi-disciplinary open access archive for the deposit and dissemination of scientific research documents, whether they are published or not. The documents may come from teaching and research institutions in France or abroad, or from public or private research centers.
L'archive ouverte pluridisciplinaire HAL, est destinée au dépôt et à la diffusion de documents scientifiques de niveau recherche, publiés ou non, émanant des établissements d'enseignement et de recherche français ou étrangers, des laboratoires publics ou privés. 


\title{
Lyapunov event-triggered control: a new event strategy based on the control
}

\author{
N. Marchand ${ }^{*, * *}$ J.J. Martinez ${ }^{*, * *}$ S. Durand ${ }^{* * *}$ \\ J.F. Guerrero-Castellanos ${ }^{* * * *}$ \\ * CNRS, GIPSA-lab, 11 rue des mathématiques, 38402 Grenoble, \\ France \\ ** Univ. Grenoble Alpes, GIPSA-lab, F-38402 Grenoble, France \\ nicolas.marchand@gipsa-lab.fr \\ *** UMI LAFMIA, CNRS - CINVESTAV, Mexico D.F., Mexico, \\ sylvain@durandchamontin.fr \\ **** Autonomous University of Puebla (BUAP), Faculty of Electronics, \\ Puebla, Mexico, fguerrero@ece.buap.mx
}

\begin{abstract}
Event-triggered control is a sampling strategy that updates the control value only when some events occur. An event is usually generated by an event-function that indicates if the control signal must be updated or not. If one excepts self-triggered implementation, event-triggered control requires the evaluation of the event function at each time instant. Unfortunately, in the literature of nonlinear system event-based control, computing the event function is more resource consuming than computing the control itself. Moreover, it requires the knowledge of a Lyapunov function that is not necessarily available. The purpose of this paper is to propose for affine nonlinear systems a new strategy for the choice of the event function that only requires the computation of the control. This reduces the complexity of computing the event and avoids to know the Lyapunov function.
\end{abstract}

Keywords: Nonlinear control, event-triggered control, Lyapunov, general formula for control

\section{INTRODUCTION}

In the past years, a new approach for sampling controlled systems appeared. It is usually called event-based control and consists in updating the control upon an event depending on the system itself, that is the state or the output, instead of depending upon the time as for classical periodic sampling. The idea was first developed by the seminal works of Åström and Bernhardsson [2002] and Årzén [1999]. In the first contribution, it was shown that with less computations, event-based control can achieve the same performance when in the second, the first event driven PID was proposed. The development of event-based control strategies started with level-crossing approaches like in [Velasco et al., 2009, 2003] or [Durand and Marchand, 2009, Durand et al., 2011]. Event-based control now usually relies on a triggering algorithm as represented in figure 1 .

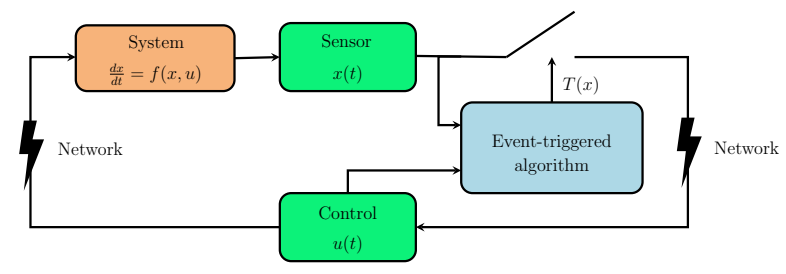

Fig. 1. Event-based control [Seuret et al., 2013]

As in [Marchand et al., 2013], the triggering algorithm usually takes the form of an event function $e: \mathcal{X} \times$
$\mathcal{X} \rightarrow \mathbb{R}$ that indicates if one needs $(e \leq 0)$ or not $(e>0)$ to update the control value. $\mathcal{X}$ represents the state space. The event function $e$ takes the current state $x$ as input and a memory $m$ of $x$ last time $e$ became negative (defined later on). In the Periodic Event-triggered Control scheme (PEC), a periodic sampling is given and the event function indicates the control must be updated at the next sampling instant [Heemels et al., 2013, 2011] whereas in the Continuous Event-triggered Control scheme (CEC), the control function is updated instantaneously after the zeroing of the event function [Anta and Tabuada, 2010, Tabuada, 2007, Anta and Tabuada, 2009, Marchand et al., 2013]. If the first approach has the advantage to guaranty a minimal sampling period (the control can not be updated more often than the a priori given period) it is not very suitable for general nonlinear systems of the form $\dot{x}=f(x, u)$ where the application of the classical periodic sampling is known to be delicate. The PEC strategy relies either on the existence of a Lipschitz stabilizing control law and an ISS-CLF, that is a Control Lyapunov Function such that $\frac{\partial V}{\partial x} f(x, k(x+\varepsilon)) \leq-\alpha(\|x\|)+\beta(\|\varepsilon\|)$ where $\alpha$ and $\beta$ are two functions of class $\mathcal{K}_{\infty}$ and $\varepsilon=m-x$ denotes the measurement error. The control is updated each time $e(x, m):=\sigma \alpha(\|x\|)-\beta(\|\varepsilon\|) \leq 0$ ensuring that way the strict decrease of the CLF with $0<\sigma<1$. Or, as in [Marchand et al., 2013] for affine nonlinear systems of the form $\dot{x}=f_{a}(x)+g_{a}(x) u$, the event function is defined relative to the derivative of the CLF as $e(x, m):=$ $\sigma \frac{\partial V}{\partial x}\left(f_{a}(x)+g_{a}(x) k(x)\right)-\frac{\partial V}{\partial x}\left(f_{a}(x)+g_{a}(x) k(m)\right)$ where here again $0 \leq \sigma<1$ is a tuning parameter. 
The triggering algorithm defined by the event function $e$ is associated to a static feedback law $k: \mathcal{X} \rightarrow \mathcal{U}$ that derives from a continuous time analysis where $\mathcal{U}$ denotes the control space.

In all the existing continuous event-triggered control strategies developed for nonlinear systems, computing the event requires to compute a function depending upon the current state value, upon the value of the state at the previous event instant and upon the control value. If the data sent over the network is lower with this approach, the complexity of the computations is much higher than with a classical continuous time implementation. For instance, for linear system with a quadratic CLF, $e$ is a quadratic polynomial in $x$ and $m$. Moreover, in all the event strategies mentioned above, the knowledge of the Lyapunov function is required to compute the event function.

The aim of this paper is to give some answers to the two above limitations. In a first step, we assume that a control stabilizing the system exists. Based on this assumption, an event function is proposed. It only requires the knowledge of the control to guaranty stability and non zero interexecution time. In a second step, we propose a new event function based on the sole control for the general eventtriggered feedback formula proposed in [Marchand et al., 2013] and derived from Sontag's general formula (see [Clarke et al., 1997] for its more general formulation). To our knowledge, event functions based on the control has never been proposed for nonlinear systems in the literature before.

\section{PRELIMINARIES}

In this paper, we focus on affine in the control dynamical systems defined by:

$$
\dot{x}=f(x)+g(x) u
$$

where $x \in \mathcal{X} \subset \mathbb{R}^{n}, u \in \mathcal{U} \subset \mathbb{R}^{p}$, and $f$ and $g$ are smooth functions with $f$ vanishing at the origin. For sake of simplicity, we only consider in this paper null stabilization with initial time instant $t_{0}=0$. If system (1) admits an asymptotic stabilizing feedback $k: \mathcal{X} \rightarrow \mathcal{U}$ then there exists a Control Lyapunov Function $V: \mathcal{X} \rightarrow \mathbb{R}$, that is a smooth function, positive definite and such that:

$$
\dot{V}=\frac{\partial V}{\partial x}[f(x)+g(x) k(x)]
$$

It is worth noting that if $k$ is assumed to be smooth, then $V$ is known to exist and to be as smooth as $k$. In the present paper, only the smoothness of $V$ is required which is a little bit less restrictive than the one of $k$.

In the following, let us use the following notations. Let

$$
a(x):=\frac{\partial V}{\partial x} f(x)
$$

and

Let us define the sets:

$$
b(x):=\frac{\partial V}{\partial x} g(x)
$$

$$
\begin{gathered}
\mathcal{S}_{b}:=\{x \in \mathcal{X} \mid\|b(x)\| \neq 0\} \\
\mathcal{S}_{a}:=\{x \in \mathcal{X} \mid a(x) \geq 0\}
\end{gathered}
$$

where $\|\cdot\|$ stand for the 2 -norm. Let $\|\cdot\|_{1}$ and $\|\cdot\|_{\infty}$ denote respectively the 2 -norm and the infinite norm. For any set, let the superscript " $*$ " be added when considering the set without $\{0\}$. Let $v_{i}$ denote for any vector $v$ its $\mathrm{i}^{\text {th }}$ component. Let $\mathcal{B}(\delta)$ denote the ball or radius $\delta>0$ centred at the origin.

We recall here the definition of semi-uniform Minimum Sampling Interval (MSI) event-triggered control:

Definition 1. [Marchand et al., 2013] An event-triggered feedback $(k, e)$ is said to be semi-uniformly MSI iff for all $\delta>0$, and all $x_{0}$ in the ball of radius $\delta$ centered at the origin $\mathcal{B}(\delta)$, the inter-execution times, that is the duration between two successive events, can be below bounded by some $\underline{\tau}(\delta)>0$.

It is known that a nonlinear system of the form (1) with a semi-uniformly MSI event-based feedback $(k, e)$, the solution of (1) starting in $x_{0} \in \mathcal{X}$ at $t=0$ is defined for all positive $t$ as the solution of the differential system:

$$
\begin{aligned}
& \dot{x}=f(x)+g(x) k(m) \\
& \left\{\begin{array}{l}
m=x \text { if } e(x, m) \leq 0, x \neq 0 \\
\dot{m}=0 \text { elsewhere }
\end{array}\right. \\
& \text { with: } x(0)=x_{0} \text { and } m(0)=x(0)
\end{aligned}
$$

\section{AN EVENT FUNCTION BASED ON THE CONTROL}

In this section, we assume first that a stabilizing feedback exists for system (1):

Hypothesis 2. There exists a feedback $k: \mathcal{X} \rightarrow \mathcal{U}$ and a smooth Lyapunov function $V: \mathcal{X} \rightarrow \mathbb{R}^{+}$such that $a(x)+b(x) k(x)<0$ for all $x \neq 0$

This assumption implies in particularly that $\mathcal{S}_{a} \subset \mathcal{S}_{b}$. In addition, we assume:

Hypothesis 3. There exists a strictly positive function $\omega$ : $\mathcal{X} \rightarrow \mathbb{R}^{+}$such that the feedback can be written as $k(x)=$ $-b^{T}(x) \omega(x)$.

Hypothesis 3 is directly linked to the existence of a smooth feedback. Indeed, coupled to hypothesis 2, it guaranties that:

$$
a(x)-b(x) b^{T}(x) \omega(x)<0
$$

which is exactly inequality (11) of [Marchand et al., 2013] that is known to ensure a smooth feedback using Sontag's general formula. Since $\omega$ is assumed to be positive, it also gives for all $\rho \geq 1$ :

$$
a(x)-\rho b(x) b^{T}(x) \omega(x)<0
$$

With the above assumptions, our first result is the following:

Theorem 4. Assume Hypotheses 2 and 3 are satisfied for some feedback law $k$, then the event-triggered feedback $(\kappa, e)$ defined by (8)-(9) is semi-uniformly MSI for any $\rho \geq 1$.

$$
\begin{aligned}
& \kappa(x):=(1+\sqrt{2}) k(x) \\
& e(x, m):= \begin{cases}\|k(x)\|^{2}-\rho p(1+\sqrt{2})\|k(m)-k(x)\|_{\infty}^{2} & \text { on } \mathcal{S}_{a} \\
1 & \text { otherwise }\end{cases}
\end{aligned}
$$

Recall that $p$ denotes the control vector size. Note also that, right after an event, $e(x, m)=\|k(x)\|^{2}=$ 
$\omega(x)^{2}\|b(x)\|^{2}>0$ for all $x \in \mathcal{S}_{a}$. This follows from inequality (6). Note also that (9) does not require the knowledge of the Lyapunov function.

\section{Proof of Theorem 4:}

The proof is divided into two steps. First, we prove that the CLF $V$ is strictly decreasing except eventually at the event instants. In a second step, we prove that the eventbased feedback $(k, e)$ is semi-uniformly MSI, that is for any ball of initial conditions, there exists a minimal interexecution time depending only upon the ball radius. Note that the proof can be restricted to $\mathcal{S}_{b}^{*}$ since outside this set, $a(x)<0$ for all non zero $x$.

Step 1: $V$ is decreasing - Let us take $\beta:=1+\sqrt{2}$. Then one can notice that $\beta$ is the solution of

$$
\beta^{2}-2 \beta-1=0
$$

Recall also that $p$ is defined as the size of the control vector. Let us assume that $e(x, m)>0$, that is for all $i$ :

$$
\|k(x)\|^{2}>\rho p \beta\left[k_{i}(m)-k_{i}(x)\right]^{2} \geq p \beta\left[k_{i}(m)-k_{i}(x)\right]^{2}
$$

because $\rho \geq 1$. Consider first the right hand side of this equation and rewrite it using hypothesis 3 as follows for any $\beta$ :

$$
\begin{aligned}
\beta^{2} b(x) b^{T}(x) \omega(x)^{2} & -\left(\beta^{2}-1\right) b(x) b^{T}(x) \omega(x)^{2} \\
& =b(x) b^{T}(x) \omega(x)^{2}=\|k(x)\|^{2}
\end{aligned}
$$

Since $V$ is a CLF for $k$, for all non zero $x$ we have $a(x)+$ $b(x) k(x)<0$ which can be written as:

$$
a(x)-b(x) b^{T}(x) \omega(x)<0
$$

Since $\omega(x)$ is assumed strictly positive for all $x$, it follows for any $\beta \geq 1$ :

$$
-\left(\beta^{2}-1\right) b(x) b^{T}(x) \omega^{2}(x) \leq-\left(\beta^{2}-1\right) \omega(x) a(x)
$$

which finally gives:

$$
\beta^{2} b(x) b^{T}(x) \omega(x)^{2}-\left(\beta^{2}-1\right) \omega(x) a(x)>p \beta\left[k_{i}(m)-k_{i}(x)\right]^{2}
$$

Multiplying left and right hand side by $b_{i}(x)^{2}$ and summing for all $i$, it gives using (10):

$b(x) b(x)^{T}\left[\beta^{2} b(x) b(x)^{T} \omega(x)^{2}-2 \beta a(x) \omega(x)\right]>p \beta \sum_{i \in\{1, \ldots, p\}} b_{i}(x)^{2}\left[k_{i}(m)-k_{i}(x)\right]^{2}$

First looking at the left hand side of this last inequality, it follows:

$$
\begin{aligned}
a(x)^{2}+ & b(x) b(x)^{T}\left[\beta^{2} b(x) b(x)^{T} \omega(x)^{2}-2 \beta a(x) \omega(x)\right] \\
& =\left[a(x)-\beta b(x) b(x)^{T} \omega(x)\right]^{2} \\
& \geq b(x) b(x)^{T}\left[\beta^{2} b(x) b(x)^{T} \omega(x)^{2}-2 \beta a(x) \omega(x)\right]
\end{aligned}
$$

The right hand side of the inequality gives using the inequality between the 1 -norm and the 2-norm:

$$
\begin{aligned}
p \beta \sum_{i \in\{1, \ldots, p\}} b_{i}(x)^{2}\left[k_{i}(m)-k_{i}(x)\right]^{2} & \\
& \geq \beta\left[\sum_{i \in\{1, \ldots, p\}}\left|b_{i}(x)\right|\left|k_{i}(m)-k_{i}(x)\right|\right]^{2} \\
& \geq \beta[b(x)(k(m)-k(x))]^{2}
\end{aligned}
$$

Combining this two last inequalities gives:

$$
[a(x)+\beta b(x) k(x)]^{2}>\beta[b(x)(k(m)-k(x))]^{2}
$$

Applying $\kappa(\cdot):=\beta k(\cdot)$ as control, this last inequality becomes

$$
|a(x)+b(x) \kappa(x)|>|b(x)(\kappa(m)-\kappa(x))|
$$

This ensures that the CLF $V$ is strictly decreasing along the event-triggered trajectory of the system:

$$
\begin{aligned}
\dot{V} & =a(x)+b(x) \kappa(m) \\
& =a(x)+b(x) \kappa(x)+b(x)(\kappa(m)-\kappa(x))<0
\end{aligned}
$$

Step 2: $(k, e)$ is uniformly MSI - The proof is identical to [Marchand et al., 2013]. It relies on the smoothness of $k$. We will not detail the proof here but we will just give the spirit of it. The proof consists in showing that when an event occurs, the next event can not happen instantaneously right after due to continuity considerations. This follows from the fact that just after an event, that is just after the control update, the event function can be lower bounded by some bound as follows:

$$
e(x, m)=\|k(x)\|^{2} \geq \underbrace{\sup _{\zeta \in \mathcal{S}_{a}}\|k(\zeta)\|^{2}}_{=: \chi\left(\vartheta_{m}\right)>0}
$$

Considering $k$ smooth, it will necessary take a non zero time $\underline{\tau}\left(\vartheta_{m}\right)$ for $e$ to vanish again.

\section{THE GENERAL FORMULA FOR FEEDBACK STABILIZATION WITH AN EVENT FUNCTION BASED ON THE CONTROL}

We focus now on the general formula for event-based control given in [Marchand et al., 2013] that ensures the existence of an event-based feedback having the semiuniform MSI property that we recall here:

Theorem 5. [Marchand et al., 2013] If there exists a CLF $V$ for system (1), then the event-based feedback $(k, e)$ defined below is semi-uniformly MSI, smooth on $\mathcal{X} \backslash\{0\}$, and such that:

$$
\frac{\partial V}{\partial x} f(x)+\frac{\partial V}{\partial x} g(x) k(m)<0, \quad x \in \mathcal{X} \backslash\{0\}
$$

where $m$ is defined in (4) and

$$
\begin{aligned}
k_{i}(x):=- & b_{i}(x) \delta_{i}(x) \gamma(x) \\
e(x, m):=- & a(x)-b(x) k(m) \\
& \quad-\sigma \sqrt{a(x)^{2}+\theta(x) b(x) \Delta(x) b(x)^{T}}
\end{aligned}
$$

where

- $x \rightarrow \Delta(x):=\operatorname{diag}\left(\delta_{1}(x), \delta_{2}(x), \ldots, \delta_{p}(x)\right)$ is a smooth function of $\mathcal{X}^{*}$ to $\mathbb{R}^{p \times p}$ strictly positive on $\mathcal{S}_{b}^{*}$

- $x \rightarrow \theta(x)$ is a smooth function of $\mathcal{X}$ to $\mathbb{R}$ positive definite on $\mathcal{S}_{b}$

- $\sigma$ is a control parameter in $[0,1[$,

- $\gamma: \mathcal{X} \rightarrow \mathbb{R}$ is defined by:

$$
\gamma(x):= \begin{cases}\frac{a(x)+\sqrt{a(x)^{2}+\theta(x) b(x) \Delta(x) b(x)^{T}}}{b(x) \Delta(x) b(x)^{T}} & \text { if } x \in \mathcal{S}_{b} \\ 0 & \text { if } x \notin \mathcal{S}_{b}\end{cases}
$$

The main problem of the control law (13-14) resides 1$)$ in the complexity that requires the evaluation of the event function, 2) in the knowledge of the Lyapunov function that is required to compute (14). As before, the aim of the following theorem is therefore to propose a simpler event function for the general formula.

Theorem 6. If there exists a CLF $V$ for system (1), then the event-based feedback $(k, e)$ is semi-uniformly MSI, 
smooth on $\mathcal{X} \backslash\{0\}$, and such that (12) is satisfied with $k$ as in (13) and $e$ defined by:

$$
e(x, m):= \begin{cases}\inf _{i \in\{1, \ldots, p\}} \theta(x) \delta_{i}(x)-p\left[k_{i}(m)-k_{i}(x)\right]^{2} & \text { on } \mathcal{S}_{a} \\ 1 & \text { otherwise }\end{cases}
$$

Note that each time an event occurs (that is, each time $e$ vanishes), right after the update of the control value, the event function becomes strictly positive: $e(x, m)=$ $\theta(x) \delta_{i}(x)>0$.

\section{Proof of Theorem 6 :}

The proof is divided in two steps as for Theorem 4. In the first one, we prove that the CLF $V$ is strictly decreasing and in a second step, we establish that the event-based feedback $(k, e)$ is semi-uniformly MSI.

Step 1: $V$ is decreasing - If $e(x, m)>0$, then we have for all $i \in\{1, \ldots, p\}$ :

and therefore

$$
\theta(x) \delta_{i}(x)>p\left[k_{i}(m)-k_{i}(x)\right]^{2}
$$

$$
b_{i}(x)^{2} \theta(x) \delta_{i}(x) \geq p b_{i}(x)^{2}\left[k_{i}(m)-k_{i}(x)\right]^{2}
$$

Note that this last inequality is strict everywhere $b(x)$ does not vanish, that is on $\mathcal{S}_{b}:=\{x \in \mathcal{X}\|\| b(x) \| \neq 0\}$. Summing this last inequality for all $i$ and writing this sum in a matrix form, it gives:

$$
\theta(x) b(x) \Delta(x) b(x)^{T} \geq p \sum_{i \in\{1, \ldots, p\}} b_{i}^{2}(x)\left[k_{i}(m)-k_{i}(x)\right]^{2}
$$

Since for all non zero $x \in \mathcal{X} \backslash \mathcal{S}$, one knows that $a(x)<0$, it follows:

$$
a^{2}(x)+\theta(x) b(x) \Delta(x) b(x)^{T}>p \sum_{i \in\{1, \ldots, p\}} b_{i}^{2}(x)\left[k_{i}(m)-k_{i}(x)\right]^{2}
$$

It gives using the inequality between the 2-norm and the 1-norm:

$$
a^{2}(x)+\theta(x) b(x) \Delta(x) b(x)^{T}>\left[\sum_{i \in\{1, \ldots, p\}} b_{i}(x)\left[k_{i}(m)-k_{i}(x)\right]\right]^{2}
$$

This last inequality finally gives:

$$
-\sqrt{a(x)^{2}+\theta(x) b(x) \Delta(x) b(x)^{T}}+b(x)(k(m)-k(x))<0
$$

Since, along the trajectory of the system, one has:

$$
\begin{aligned}
\dot{V} & =a(x)+b(x) k(m) \\
& =a(x)+b(x) k(x)+b(x)(k(m)-k(x)) \\
& =-\sqrt{a(x)^{2}+\theta(x) b(x) \Delta(x) b(x)^{T}}+b(x)(k(m)-k(x))
\end{aligned}
$$

it follows that, as long as $e(x, m)>0$, the time derivative of the CLF $V$ remains strictly negative.

Each time an event occurs (that is, each time $e$ vanishes), right after the update of the control value, $e(x, m)=$ $\theta(x) \delta_{i}(x)>0$. Therefore, the CLF can vanish only "punctually" at the instants when $e$ vanishes. At all other instants, the CLF is strictly decreasing.

Step 2: $(k, e)$ is uniformly MSI - The proof is identical to the one in [Marchand et al., 2013].

\section{THE LINEAR CASE}

Consider a linear system given by:

$$
\dot{x}=A x+B u
$$

and $P$, the solution of the Ricatti equation:

$$
P A+A^{T} P-2 \varepsilon P B B^{T} P=-Q
$$

where $Q$ is a symmetric definite positive. Taking $V=$ $x^{T} P x$ as CLF, it follows

$$
\begin{aligned}
& a(x)=x^{T}\left(P A+A^{T} P\right) x \\
& b(x)=2 x^{T} P B
\end{aligned}
$$

Taking $\Delta(x)=I_{n}$ and $\theta(x)=\varepsilon^{2} b(x) b(x)^{T}-2 \varepsilon a(x)$, the feedback law (13) becomes using (18):

$$
k(x)=-\varepsilon b(x)^{T}=\underbrace{-2 \varepsilon B^{T} P}_{=: K} x
$$

Rewriting $\theta(x)$, one has:

$$
\begin{aligned}
\theta(x) & =\varepsilon^{2} b(x) \Delta(x) b(x)^{T}-2 \varepsilon a(x) \\
& =2 \varepsilon x^{T} Q x
\end{aligned}
$$

Therefore, applying Theorem 6 gives the event function

$$
e(x, m):=2 \varepsilon x^{T} Q x-p \sup _{i \in\{1, \ldots, p\}}\left[k_{i}(m)-k_{i}(x)\right]^{2}
$$

Applying this event-triggered strategy therefore requires to compute the current control value $k(x)$ (event if not applied) and the corresponding Lyapunov function. At the opposite, applying Theorem 4 only requires the computation of $k(x)$ and gives for $\rho=1$ :

$e(x, m):=\|k(x)\|^{2}-p(1+\sqrt{2}) \sup _{i \in\{1, \ldots, p\}}\left[k_{i}(m)-k_{i}(x)\right]^{2}$

If this event function is positive, it follows for all $i \in$ $\{1, \ldots, p\}$ :

$$
p(1+\sqrt{2})\left(k_{i}(x)-k_{i}(m)\right)^{2}<k(x)^{T} k(x)
$$

This last inequality can be further transformed:

$$
p(1+\sqrt{2})\|k(x)-k(m)\|_{\infty}^{2}<\|k(x)\|^{2}
$$

Introducing the classical inequalities between norms, if the following inequality holds, then (21) also holds:

$$
(1+\sqrt{2})\|k(x)-k(m)\|^{2}<\|k(x)\|^{2}
$$

Let $\Upsilon$ denote the principal square root of $K^{T} K$. Since $K^{T} K$ is symmetric semi-definite positive, $\Upsilon$ is uniquely defined and symmetric semi-definite positive [Koeber and Schfer, 2006]. Inequality (22) becomes the following linear matrix inequality:

$$
(x-m)^{T} \Upsilon^{T} \Upsilon(x-m)<\frac{1}{1+\sqrt{2}} x \Upsilon^{T} \Upsilon x
$$

Note that deriving the event function from (21) instead of (22) can be computationally less consuming.

\section{NUMERICAL EXAMPLE}

We consider here the nonlinear system proposed in [Anta and Tabuada, 2008, Marchand et al., 2013]:

$$
\begin{aligned}
& \dot{x}_{1}=-x_{1}^{3}+x_{1} x_{2}^{2} \\
& \dot{x}_{2}=x_{1} x_{2}^{2}-x_{1}^{2} x_{2}+u
\end{aligned}
$$

This system is known to admit $V(x)=\frac{1}{2} x_{1}^{2}+\frac{1}{2} x_{2}^{2}$ as CLF with $a(x)=-x_{1}^{4}+x_{1} x_{2}^{3}, b(x)=x_{2}$. Therefore, using Theorem 6 gives a first event-triggered control law for instance taking $\sigma=0.9, \Delta=I$ and $\theta(x)=\|b\|^{2}$.

Defining $\omega$ as follows:

$$
\omega(x)=x_{1} x_{2}+\frac{1}{2} x_{1}^{2}+\frac{1}{2} x_{2}^{2}
$$

it is also known that the control $k(x):=-b(x)^{T} \omega(x)$ globally asymptotically stabilizes system (24). Therefore, 
Hypotheses 2 and 3 are satisfied. Hence, Theorem 4 gives a second event-based control.

In order to test the proposed event-triggered control, we compared them with the event-triggered discontinuous control proposed [Marchand et al., 2013] with an event function based on the time derivative of the CLF and with [Anta and Tabuada, 2008] with an event function based on the ISS property of the system. The result is presented in Figure 2. The new event strategy looks parsimonious like the general formula was. The impact of $\rho$ in Theorem 4 on the performance seems to be limited. Increasing it seems to reduce the number of event. The simulation where carried out taking $\rho=10$

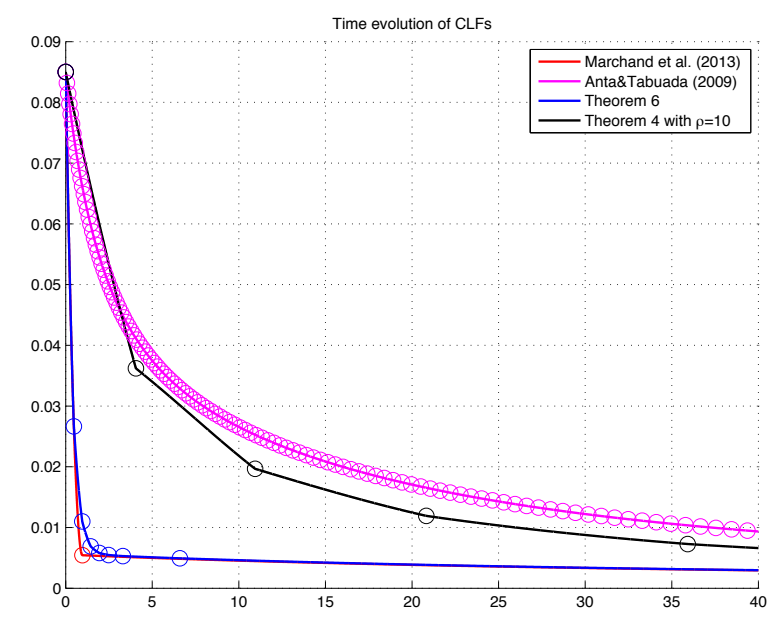

Fig. 2. System (24) under different event-triggered control strategies. The circles emphasize events, that is an update of the control law.

\section{CONCLUSION}

In this paper, we have proposed two different eventtriggered feedback laws for control-affine nonlinear systems. Contrary to the feedback law existing in the literature, computing the proposed feedback and event function is of the same complexity level as computing the sole feedback. Moreover, the triggering strategies are not based on the Lyapunov function that is no more required (at least for Theorem 4).

\section{REFERENCES}

A. Anta and P. Tabuada. Self-triggered stabilization of homogeneous control systems. In Proc. IEEE American Control Conf. (ACC), pages 4129-4134, 2008.

A. Anta and P. Tabuada. Isochronous manifolds in selftriggered control. In Proceedings of the 48th IEEE Conference on Decision and Control, 2009 held jointly with the 2009 28th Chinese Control Conference, pages 3194-3199, 2009.

A. Anta and P Tabuada. To sample or not to sample: Selftriggered control for nonlinear systems. IEEE Trans. on Automatic Control, 55:2030 - 2042, 2010.

K. E. Årzén. A simple event based PID controller. In Proceedings of the 14th IFAC World Congress, Prague, Czech republic, 1999.
K. J. Åström and B. M. Bernhardsson. Comparison of Riemann and Lebesgue sampling for first order stochastic systems. In Proceedings of the 41st IEEE Conference on Decision and Control, volume 2, pages 2011-2016, 10-13 Dec. 2002.

F. H. Clarke, Y. S. Ledyaev, E. D. Sontag, and A. Subbotin. Asymptotic controllability implies feedback stabilization. IEEE Trans. on Automatic Control, 42(10): 1394-1407, 1997.

S. Durand and N. Marchand. Further results on eventbased PID controller. In Proceedings of the European Control Conference (ECC), Budapest, Hungary, 2009.

S. Durand, N. Marchand, and J. F. Guerrero-Castellanos. Simple Lyapunov sampling for event-driven control. In Proceedings of the IFAC World Congress, Milano, Italy, 2011.

W. Heemels, MCF Donkers, and AR Teel. Periodic event-triggered control based on state feedback. In 50th IEEE Conference on Decision and Control and European Control Conference (CDC-ECC), pages 25712576. IEEE, 2011.

W. Heemels, M. Donkers, and A. Teel. Periodic eventtriggered control for linear systems. IEEE Trans. on Automatic Control, 58(4):847-861, 2013. doi: 10.1109/ TAC.2012.2220443.

Martin Koeber and Uwe Schfer. The unique square root of a positive semidefinite matrix. International Journal of Mathematical Education in Science and Technology, 37(8):990-992, 2006. doi: 10.1080/ 00207390500285867. URL http://www.tandfonline. com/doi/abs/10.1080/00207390500285867.

N. Marchand, S. Durand, and J. F. Guerrero-Castellanos. A general formula for event-based stabilization of nonlinear systems. IEEE Trans. on Automatic Control, 58(5), 2013. doi: 10.1109/TAC.2012.2225493. URL http://hal archives-ouvertes.fr/hal-00675946/.

A. Seuret, C. Prieur, and N. Marchand. Stability of nonlinear systems by means of event-triggered sampling algorithms. Journal of Mathematical Control and Information, 2013. To appear.

P. Tabuada. Event-triggered real-time scheduling of stabilizing control tasks. IEEE Trans. on Automatic Control, 52(9):1680-1685, 2007.

M. Velasco, P. Martí, and J. M. Fuertes. The self-triggered task model for real-time control systems. In Work-inProgress Session of the 24th IEEE Real-Time Systems Symposium, 2003.

M. Velasco, P. Martí, and E. Bini. On Lyapunov sampling for event-driven controllers. In Proc. IEEE Int. Conf. on Decision and Control (CDC), 2009. 